\title{
A joint optimization strategy for scale-based product family positioning
}

\author{
Yangjian Ji*, Tianyin Tang, Chunyang Yu, Guoning Qi \\ Industrial Engineering Center, Department of Mechanical Engineering, \\ Zhejiang University, Hangzhou 310027, China
}

Received 1 November 2013

Accepted 6 March 2014

\begin{abstract}
With the development of modern technologies and global manufacturing, it becomes more difficult for companies to distinguish themselves from their competitors. In order to keep their competitive advantages, companies must properly position their product families by offering a right product portfolio to each target market. To evaluate competitive advantages for a scale-based product family, this paper takes product family competitive advantage (PFCA) as a measure metric which is consisted of customer choice probability, sales, and profit. Meanwhile, to keep lower manufacturing costs, a commonality index of scale-based product family is proposed based on product design technology parameters in a product family. A multi-objective joint optimization model that balances the competitive advantages and the commonality is proposed. Based on a case study of motor product family positioning, Pareto frontier solutions are generated by genetic algorithm, and the results show that the joint optimization model excels in supporting product family positioning.
\end{abstract}

Keywords: Scale-based product family, product portfolio, product family positioning, joint optimization

\section{Introduction}

Many companies turn to mass customization in response to the tremendous changes in today's global marketplace [1][2], due to the fact that mass customization is able to satisfy individual customer requirements with the efficiency of mass production [3][4]. Several strategies have been proposed to help producers to build mass customized products and maintain a lower cost. Among these, product family strategy is one of the most popular operations management approaches, in which each product has unique functions or features to meet specific customer requirements [5][6]. To stimulate sales and maximize revenue, manufacturers intend to provide a variety of product variants in a product family [5]. However, high variety will result in inefficiencies in manufacturing, and accordingly the costs increase exponentially [7]. In this regard, it is important for a company to resolve the tradeoffs between product diversity and engineering costs [8].
Generally, it is believed that satisfying more market requirements will bring more sales and accordingly more profits. Therefore, a company often assesses to what extent one product variant is able to satisfy market requirements, and then determine an optimal portfolio to satisfy as many market requirements as possible. However, based on a marketing analysis report by Simpson et al. [9], meeting more market requirements is not always optimal for a small or medium-sized company. Although its products may be equally possible to satisfy customer requirements of individual market segments, it will have a smaller market share and fewer profits because of its weaker product competition ability. Based on the theory of competitive intensity, targeting some specific market segments by satisfying their customers may help gain competitive advantages and increase sales and profits. Accordingly, it is more reasonable that the product family competitive advantage (PFCA) should be regarded as a metric during the optimization of product diversity.

\footnotetext{
* Corresponding Author. Tel.: +86-571-87953242 E-mail: mejyj@zju.edu.cn 
To control engineering costs, increasing commonality of a product family has been widely considered as an effective method. Commonality can be obtained by minimizing the non-value added variations across the product variants within a family without limiting the choices of customers in each market segment, i.e., make each product within a family distinct in ways customers notice and identical in ways customers cannot see [11]. High commonality means reduced product line complexity, decreased setup and retooling time, and increased productivity [10]. However, if commonality is too high, variants lack distinctiveness and individual performance of products of a family is not optimized. PFCA will accordingly be low, and vice versa [11]. Therefore, PFCA and commonality need to be well leveraged by proper positioning, and the relevant tradeoff analysis between PFCA and commonality essentially necessitates a formulation of joint optimization of both PFCA and commonality.

There are two types of product families: module-based product family and scale-based product family. Accordingly, there are two kinds of metrics of PFCA and commonality. For the module-based product family, product diversity is derived by adding, substituting, and/or removing one or more functional modules [12][13]. The positioning method of module-based product family is essentially a combinatorial optimization problem of various modules to tradeoff PFCA and commonality. However, the product diversity of scale-based product family is obtained by "stretching" or "shrinking" scaling parameters of a product in one or more dimensions [14]. As a result, the positioning method of scale-based product family is essentially a combinatorial optimization problem of various parameters to tradeoff PFCA and commonality. This paper takes scale-based product family as the research objective and focuses on the issue of product family positioning using a joint optimization method. The rest of the paper proceeds as follows. Section 2 reviews related work regarding product family positioning. Customer requirements, market segments, and parameters in product family are described in Section 3. Metrics of PFCA and commonality index are established in Section 4 and Section 5, respectively. Section 6 elaborates the optimization model for product family positioning and develops a genetic algorithm (GA) for multi-objective optimization. A case study is reported in Section 7. The paper concludes in Section 8 with discussions on further research.

\section{Related Work}

\subsection{Evaluation metrics}

Product family design involves the challenges of how to balance the commonality of the products in the family and individual performance (i.e., distinctiveness) of each product in the family [15]. Both commonality and performance need a set of properly designed metrics for an evaluation purpose. In many cases, such metrics focus on two pillars: commonality and revenue.

The degree of commonality index proposed by Collier [10] was one of the first such indices. It uses information contained in the company's bills of materials to assess commonality of a single end item, a product family, or an entire product line. Jiao and Tseng [16] extended Collier's commonality index and created indices for component part commonality and process commonality. Martin and Ishii [17] also introduced a commonality index similar to Collier's, along with indices for measuring setup costs and product differentiation points, which correlate with many of the indirect costs caused by variety. For measuring the degree of variation within a scale-based product family, the non-commonality index (NCI) was proposed by Simpson et al. [18], which is a normalized measure of the variability of design variable setting across members of the product family. A smaller NCI indicates less variation among design variable settings across the family, and, hence, more commonality within the family. Based on a similar principle to that of the NCI, Tian [19] proposed an exponential coefficient to evaluate the similarity of two design parameter vectors and builds a commonality index based on this similarity.

Another category of metrics is to measure revenue. For example, $\mathrm{Li}$ and Azarm [20] adopted the Net Present Value (NPV) in economics to evaluate the benefits of the product family in order to maximize revenue. de Week et al. [21] introduced the sales volume of a product family by comparing it with the benchmark products which have the maximum sales volume. Pullmana et al. [22] took sales volume as an optimization objective and a conjoint analysis was used to assess product characteristics. To balance the benefits of customers and of producers, Jiao et al. [23] propose a concept of shared surplus taking customer preference, customer choice probability, size of the market segments, and cost. However, all these metrics assume that the predominated market is known a priori, and the revenue is normally unrealistic under the competitive circumstances. Taking the competitive products in the market as a bench- 
mark, the metrics of PFCA and commonality are proposed in the paper.

\subsection{Optimization approaches}

Product family positioning essentially entails a type of multi-objective optimization problems [9]. When using multi-objective optimization to determine the best design variable settings for individual products within a product family, there are two basic optimization approaches: two-step approach and single-step approach [24]. The two-step approach divides the optimal process into two steps: the entire alternative product variants are enumerated in the first step, and then promising products are selected to constitute a product portfolio in the second step [9]. Following the two-step approach, Green and Krieger [25][26] introduced several heuristic procedures with the consideration of how to generate a reference set appropriately. In general, the two-step approach is preferred only when the number of alternatives is small and product attributes are simple [9]. The single-step approach uses a single optimized computation for product family planning based on internal and external characteristics directly. An example of the single-step approach was proposed by Fujita et al. [27] who simultaneously optimized the system structure and configuration of a product family and applied their approach to a family of aircraft. In general, the one-step approach is more preferable when the number of alternatives is large, as the intermediate step of enumeration can be eliminated [28].

\subsection{Optimization algorithms}

Product family positioning is classified as a combinatorial optimization problem, in that each company strives for the optimality of its product offerings through various combinations of products [29]. By intuition, finding the near-optimal solution for a finite combinatorial optimization problem could be done by simple enumeration. In practice, however, this technique is often impossible because the number of feasible solutions is often enormous. A number of methods and algorithms have been developed to solve combinatorial optimization problems. Sait and Youssef [30] divided them into two groups: exact algorithms and approximation algorithms. Due to the enumerative nature, exact algorithms are not easy to design with moderate computational effort as can be seen from the complexity theory [31]. Comparing with traditional calculus-based or approximation-based optimization techniques, GAs excel in solving combinatorial optimization problems [32]. However, GAs are in general incapable of fine-tuning for obtaining the global optimum [33]. As a result, various modified GAs have been proposed for particular problems [34]. For example, Jiao et al. [23] used a new coding method and a multi-gene crossover operator. Yang et al. [35] proposed a hybrid algorithm which is a combination of GA and neural network algorithm.

\section{Problem Description}

This paper aims to deal with the problem of scale-based product family positioning. We will build an optimization model with the goal of finding the optimal product combination among a set of alternative product variants. The optimal product portfolio, considering the tradeoff between PFCA and commonality, constitutes the expected product family.

The parameters of a product family can be divided into three facets: basic requirement parameter (BRP), performance requirement parameter (PRP) and design technology parameter (DTP). BRPs describe a basic function or performance of a product variant, by which the market is divided into several market segments. PRPs reflect characteristics of products that are relevant to customer experience or expectation. To some extent, PRPs can determine the level of customer satisfaction. DTPs refer to parameters which engineers are interested in. For example, when customers want to buy a car, features of style, fuel type and engine power can be considered as BRPs, fuel consumption per kilometer, maximum speed, and price as PRPs, and diameter of axle, gear number of engine as DTPs.

BRPs can be defined as $R=\left\{r_{t} \mid 1 \leq t \leq T\right\}$, where $T$ is the total number of parameters in BRPs. For each $r_{t}\left(\forall r_{t} \in R\right)$, its value is composed of a series of discrete values or ranges, i.e. $r_{t}^{*}=\left\{r_{t l}^{*} \mid 1 \leq l \leq L_{t}\right\}$. For each $r_{t}^{*}$, $L_{t}$ may be different. According to different value combinations of $r_{t}$, the whole market $M$ can be divided into $I$ market segments, where $M=\left\{m_{1}, \cdots, m_{i} \cdots, m_{I}\right\}, 1 \leq I \leq \prod_{t=1}^{T} L_{t}$ and $\forall m_{i} \in M, \quad m_{i}=\left\{r_{1 i}^{*}, \cdots r_{t i}^{*}, \cdots r_{T i}^{*}\right\}$.

For $I$ market segments, we assume that there are also $I$ corresponding alternative products in a company to be positioned, i.e. $P=\left\{P_{i} \mid 1 \leq i \leq I\right\}$, and $P_{i}\left(\forall P_{i} \in P\right)$ can meet the corresponding market segment $m_{i}$ ( $\forall m_{i} \in M \quad$ ). Furthermore, for each $P_{i}$ $\left(\forall P_{i} \in P\right)$, there are a number of $\left(N_{i}-1\right)$ competitive products in the market segment, and $N_{i}$ may be different for different product $P_{i}$. 
PRPs can be defined as $G=\left\{g_{z} \mid 1 \leq z \leq Z\right\}$, where $\mathrm{Z}$ is the total number of variables in PRPs. For $N_{i}$ products in the same market segment, although the values of BRPs are the same, the values of PRPs may be different because of different technology or economic factors. The value of $g_{z}\left(g_{z} \in G\right)$ is composed of a series of discrete values or ranges, i.e. $g_{z}^{*}=\left\{g_{z k}^{*} \mid 1 \leq k \leq K_{z}\right\}$. Among the $K_{z}$ values, there are always a maximum value $g_{z}{ }^{* \max }$ and a minimum value $g_{z}^{* \min }$. For product $P_{i}$, its values of $g_{z i}^{*}$ make a major impact on the product competitive advantage.

On the other hand, a number of $S$ DTPs can be formalized as $D=\left\{d_{s} \mid 1 \leq s \leq S\right\}$. For each $d_{s}\left(\forall d_{s} \in D\right)$, its value is composed of a series of discrete values or ranges, i.e. $d_{s}^{*}=\left\{d_{s v}^{*} \mid 1 \leq v \leq V_{s}\right\}$. For each $d_{s}^{*}$ in product family $P, V_{s}$ may be different. To improve commonality of product family $P$, it is necessary to control the value of $V_{s}$.

According to the PRPs and DTPs, a decision variable $X=\left\{x_{1}, \cdots x_{i}, \cdots x_{I}\right\}$ can be defined to describe which products are selected as the members of product family by a company. $x_{i}$ $\left(x_{i} \in X\right)$ is equal to 1 or 0 , where 1 means the product $P_{i}$ is selected, and vice versa.

\section{Metric of PFCA}

\subsection{Choice probability}

When a company cannot monopolize a market segment, products of a company will inevitably face competition with products in the same category from other companies. The competition often leads to the fact that a product with satisfied BRPs will not be necessarily purchased by customers. Customer choices also depend on the values of PRPs, which reflect the level of customer satisfaction.

\subsubsection{Measurement of customer satisfaction}

For the variable value $g_{z i}^{*}$ of the product variant $p_{i}$, its satisfaction level $u_{z i}(1 \leq z \leq Z, 1 \leq i \leq I)$ can be calculated as follows:

(i) If customer satisfaction is more preferable when the values of PRPs increase (the-more-the-better), $\quad u_{z i}$ is calculated as follows:

$$
u_{z i}=\frac{g_{z i}^{*}-g_{z}^{{ }^{* \min }}}{g_{z}^{* \max }-g_{z}^{* \min }}
$$

(ii) If customer satisfaction is more preferable when the values of PRPs decrease (the-smaller-the-better), $u_{z i}$ is calculated as follows:

$$
u_{z i}=\frac{g_{z}^{{ }^{*} \max }-g_{z i}^{*}}{g_{z}^{* \max }-g_{z}^{* \min }}
$$

Here, $u_{z i} \in[0,1] . u_{z i}=1$ means that $g_{z i}^{*}$ is the benchmark among $N_{i}$ products and possesses a maximum satisfaction level.

For PRPs with $\mathrm{Z}$ variables, the customer satisfaction level $U_{i}$ of product $p_{i}$ can be evaluated as follows:

$$
U_{i}=\sum_{z=1}^{Z} q_{z} u_{z i}
$$

where $q_{z}$ is the weight of $g_{z i}$, and $\sum_{z=1}^{Z} q_{z}=1$.

\subsubsection{Measurement of choice probability}

Customer choice probability indicates how likely a customer in market $i$ chooses a product among $N_{i}$ competing products. According to the multinomial logit model [36], the probability $C_{i}$ of customers choosing the product variant $p_{i}$ can be calculated as follows:

$$
C_{i}=\frac{e^{\lambda U_{i}}}{e^{\lambda U_{i}}+\sum_{n=1}^{N_{i}-1} e^{\lambda U_{n}}}
$$

In Eq.(4), $\lambda$ is a scaling parameter. When $\lambda \rightarrow \infty$, the model is similar to the deterministic model, which means customers are absolutely rational, and the product will be selected according to the values of PRPs. However, the model becomes a uniform distribution as $\lambda \rightarrow 0$, which indicates the products will be randomly selected. The value of $\lambda$ depends on the actual market analysis.

\subsection{Holistic measurement of PFCA}

PFCA evaluates the ability of a product family bringing profits to a company by covering multiple market segments. Higher PFCA means higher probability of obtaining expected profit in a competitive circumstance. PFCA is related to the profit ratio of each variant $p_{i}$ and market size, in addition to the choice probability of each product. PFCA can be calculated holistically as follows:

$$
\begin{aligned}
\operatorname{PFCA}(X) & =I \cdot \sum_{i=1}^{I} w_{i} C_{i} o_{i} x_{i} \\
& =I \cdot \sum_{i=1}^{I} w_{i} \frac{e^{\mu U_{i}}}{e^{\mu U_{i}}+\sum_{n=1}^{N_{i}-1} e^{\mu U_{n}}} o_{i} x_{i}
\end{aligned}
$$


In Eq.(5), $w_{i}$ is the weight of market segment $i$, and $\sum_{i=1}^{I} w_{i}=1$. A larger $w_{i}$ means that market segment $i$ has more customers, and accordingly, the market size is larger. $o_{i}$ is the profit ratio of product $p_{i}$, and $\sum_{i=1}^{I} o_{i}=1$. A larger $o_{i}$ represents a higher profit. For a company, when assuming the products are mature, each $o_{i}$ is essentially invariable. $x_{i}$ ( $\left.x_{i} \in X\right)$ is a decision variable as described in Section 3.

Also, from Eq.(5), it suggests that there are some ways to improve PFCA: (1) Holding more product variants in the product family, and it is possible to cover more market segments; (2) The products should be selected if they satisfy a large number of potential customers at a higher level; (3) The products in the product family should be higher profit ratios, and can bring more profits as a whole.

\section{Commonality Index}

Higher commonality means more similar technologies will be applied in a product family, and accordingly reduces the product life-cycle cost. For a scale-based product family, commonality reflects the sharing and reusing degree of design technology parameter values among the product variants. The steps of calculating commonality are as follows:

Step1: Analyzing the aggregation degree of design technology parameter value in alternative products. If $\forall d_{s v}^{*} \in d_{s}^{*}\left(1 \leq s \leq S, 1 \leq v \leq V_{s}\right)$, there are $n_{s v}\left(1 \leq n_{s v} \leq I\right)$ same variants in a company, then the aggregation degree of $d_{s}$ $\left(\forall d_{s} \in D\right)$ can be calculated as follows:

$$
A_{s}=\sum_{v=1}^{V_{s}}\left(\frac{n_{s v}}{I^{\prime}}\right)^{2}
$$

where, $I^{\prime}=\sum_{i=1}^{I} x_{i}$ and $\sum_{v=1}^{V_{s}} n_{s v}=I^{\prime}$.

Step2: Assessing the weights of all DTPs using pair-wise comparison matrix. To evaluate the importance degree of DTP, grade criteria are shown in Table 1.

To reduce subjectivity and uncertainty, the weight of each design technology parameter can be computed by pair-wise comparison. According to Table 1, a standard pair-wise comparison matrix was established as shown in Table 2. In the matrix, if the relative importance of the $i$-th parameter to the $j$-th parameter is $e_{i j}$, then the relative importance of the $j$-th parameter to the $i$-th parameter is $e_{j i}=1 / e_{i j}$.

Table 1. Grade criteria of DTPs

\begin{tabular}{cl}
\hline Value & \multicolumn{1}{c}{ Importance degree } \\
\hline 1 & same \\
2 & Between "same" and "slightly higher" \\
3 & Slightly higher \\
4 & Between "slightly higher" and \\
& "obviously higher" \\
5 & Obviously higher \\
6 & Between "obviously higher" and \\
& "absolutely higher" \\
7 & Absolutely higher \\
8 & Between "absolutely higher" and \\
9 & "extremely higher" \\
\hline
\end{tabular}

Table 2. Standard pair-wise comparison matrix

\begin{tabular}{cccccccc}
\hline & $d_{1}$ & $\cdots$ & $d_{i}$ & $\cdots$ & $d_{j}$ & $\cdots$ & $d_{S}$ \\
\hline$d_{1}$ & 1 & $\cdots$ & $e_{1 i}$ & $\cdots$ & $e_{1 j}$ & $\cdots$ & $e_{1 S}$ \\
$\cdots$ & $\ldots$ & $\cdots$ & $\cdots$ & $\cdots$ & $\cdots$ & $\cdots$ & $\cdots$ \\
$d_{i}$ & $1 / e_{1 i}$ & $\cdots$ & 1 & $\cdots$ & $e_{i j}$ & $\cdots$ & $e_{i S}$ \\
$\cdots$ & $\cdots$ & $\cdots$ & $\cdots$ & $\cdots$ & $\cdots$ & $\cdots$ & $\cdots$ \\
$d_{j}$ & $1 / e_{1 j}$ & $\cdots$ & $1 / e_{i j}$ & $\cdots$ & 1 & $\cdots$ & $e_{j S}$ \\
$\cdots$ & $\cdots$ & $\cdots$ & $\cdots$ & $\cdots$ & $\cdots$ & $\cdots$ & $\cdots$ \\
$d_{S}$ & $1 / e_{1 S}$ & $\cdots$ & $1 / e_{i S}$ & $\cdots$ & $1 / e_{j S}$ & $\cdots$ & 1 \\
\hline
\end{tabular}

Next, the weight value $\alpha_{s}(1 \leq s \leq S)$ is determined by normalizing the elements of the eigenvector corresponding to the maximum eigenvalue calculated from the pair-wise comparison matrix [37]. Using the eigenvector assures consistency of the matrix and reduces inconsistency between the comparison values. These parameter weights will be employed during the final calculation of commonality.

Step3: according to the weight of design technology parameter and aggregation degree of its value, the commonality of the product family CI can be calculated as follows:

$$
C I=\sum_{s=1}^{S} \alpha_{s} A_{s}=\sum_{s=1}^{S}\left(\alpha_{s} \sum_{v=1}^{V_{s}}\left(\frac{n_{s v}}{I^{\prime}}\right)^{2}\right)
$$

\section{Positioning Optimization}

\subsection{Optimization model}

In order to tradeoff PFCA and commonality, we combine the Eq.(5) and Eq.(7), and a two-objective optimization model is formulated as Eq.(8). In the optimization model, the first optimization objective function is to determine the maximum value of PFCA, and the second one is to find the maximum commonality of the product family. 


$$
\begin{aligned}
& \max P F C A(X)=\max \left(I \cdot \sum_{i=1}^{I} w_{i} \frac{e^{\mu U_{i}}}{e^{\mu U_{i}}+\sum_{n=1}^{N_{i}-1} e^{\mu U_{n}}} v_{i} x_{i}\right. \\
& \max C I(X)=\max \left(\sum_{s=1}^{s}\left(\alpha_{s} \sum_{v=1}^{V_{s}}\left(\frac{n_{s v}}{I^{\prime}}\right)^{2}\right)\right) \\
& \text { s.t. } X=\left[x_{1}, \ldots, x_{i}, \ldots, x_{I}\right] \\
& I^{\prime}=\sum_{v=1}^{V_{s}} n_{s v} \\
& I^{\prime}=\sum_{i=1}^{I} x_{i} \\
& \sum_{i=1}^{I} x_{i} \geq 2 \\
& x_{i} \in\{0,1\}(i \leq I)
\end{aligned}
$$

\subsection{Optimization algorithm}

For the multi-objective optimization problem, it is reasonable to find a Pareto Solution Set (PSS) instead of an optimal solution [38]. In order to do so, a GA is proposed. The basis of GA is about representation of the problem to be solved with a finite-length string called a chromosome. For product family positioning, the length of the string stands for the total number of alternative products in the product family. Each element of the string indexed by " 1 " or " 0 ", called gene, indicating whether the product is selected or not.

Fig. 1 summarizes the procedures of the optimization solution, as elaborated below:

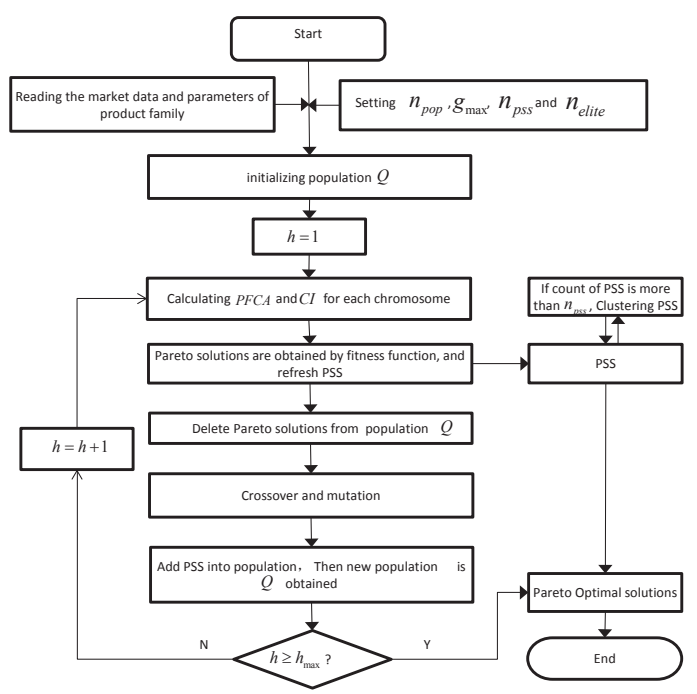

Fig. 1 procedures of optimization solution

Step1: Reading the market and parameters of the product family, such as the distribution of customer requirements, customer choice probability, profitability ratio of each alternative product variant, and so on. At the same time, solution parameters are given, such as the size of population $\left(n_{p o p}\right)$, maximum iteration loops $\left(h_{\max }\right)$, the maximum size of PSS $\left(n_{p s s}\right)$, the maximum number of Pareto solutions $\left(n_{\text {elite }}\right)$ and so on;

Step2: Generate randomly initial population $Q$ consisted of $n_{p o p}$ chromosomes. Set PSS as NULL and iteration loop $h$ is 1 ;

Step3: Calculate PFCA and $C I$ for each chromosome.

Step4: To find all non-dominated solutions in various GA search directions, a fitness function with a random weight is developed to evaluate these solutions [39]. The weights for PFCA and CI can be calculated as $q_{1} /\left(q_{1}+q_{2}\right)$ and $q_{2} /\left(q_{1}+q_{2}\right)$, respectively, where $q_{1}$ and $q_{2}$ are a random numbers in the interval $[0,1]$. As a result, the fitness degree $b_{k}$ of the $k$-th solution (i.e. the $k$-th chromosome) can be calculated as Eq.(9):

$$
b_{k}=\frac{q_{1}}{q_{1}+q_{2}} P F C A_{k}+\frac{q_{2}}{q_{1}+q_{2}} C I_{k}
$$

where $P F C A_{k}$ and $C I_{k}$ are the respective objective values in the optimization model for the $k$-th solution.

According to Eq.(9), the number of $n_{\text {elite }}$ Pareto solutions can be obtained.

Step5: Delete the Pareto solutions from population $Q$, and insert these Pareto solutions into PSS. If the count of PSS is more than $n_{p s s}$, apply the clustering method to keep the size of PSS [40].

Step6: Do the crossover and mutation operator for population $Q$ with the probability of $n_{c}$ and $n_{m}$, respectively.

Step7: Add PSS to the population, and a new population $Q$ is generated. $h=h+1$. If $h \geq h_{\max }$, then end the process. Otherwise, go to step3.

\subsection{Comprehensive optimal selecting}

For a multi-objective optimization problem, there is a contradiction trend between several objectives. The fuzzy set theory is used in this paper to evaluate all the Pareto solutions and the comprehensive optimal solution for all the objectives are then selected [41]. For the two objectives of solution $k$ in PPS, their normalization value $\mu_{P F C A}^{k}$ and $\mu_{C I}^{k}$ can be defined as Eq. (10) and (11):

$$
\mu_{P F C A}^{k}=\frac{F_{P F C A}^{k}-F_{P F C A}^{\min }}{F_{P F C A}^{\max }-F_{P F C A}^{\min }}
$$




$$
\mu_{C I}^{k}=\frac{F_{C I}^{k}-F_{C I}^{\min }}{F_{C I}^{\max }-F_{C I}^{\min }}
$$

in which $F_{P F C A}^{\max }, F_{C I}^{\max }, F_{P F C A}^{\min }$ and $F_{C I}^{\min }$ are the respective maximum and minimum values for the two objective functions in the optimization model. $F_{P F C A}^{k}$ and $F_{C I}^{k}$ are the respective objective function values for the $k$-th solution in PPS. For the solution $k$, its comprehensive normalization value $\mu^{k}$ can be evaluated as Eq. (12):

$$
\mu^{k}=\frac{\mu_{P F C A}^{k}+\mu_{C I}^{k}}{\sum_{k=1}^{N_{p s s}}\left(\mu_{P F C A}^{k}+\mu_{C I}^{k}\right)}
$$

in which $N_{p s s}$ is the count of PSS. The solution which has the maximum $\mu^{k}$ is the comprehensive optimal solution. If the solutions in PSS are sorted by $\mu^{k}$, we can get the priorities of the solutions. This will provide a basis for the decision-maker.

\section{Case Study}

Three-phase asynchronous motor is used as a case to illustrate product family positioning optimization problem. It is widely used in industry because of its simple-structure, easy maintenance, high reliable operation and low cost.

\subsection{Market requirements}

When customers choose a three-phase asynchronous motor, the power and the speed of the motor are often taken into account for decision making. Accordingly, BRPs can be defined as $R=\{$ Rated Power, Rotation Speed , and rated power and rotation speed are divided into the following discrete values:

$$
\begin{gathered}
\text { Rated Power: } r_{1}^{*}=\{3 \mathrm{~kW}, 7.5 \mathrm{~kW}, 15 \mathrm{~kW}, 22 \mathrm{~kW}, 30 \mathrm{~kW}\} \\
\text { Rotation Speed: } r_{2}^{*}=\{[3000,2800],[1500,1400],[1000,950],[750,700]\}
\end{gathered}
$$

According to the BRPs, the market can be

Table 3. The segments and their market shares divided into 20 market segments as listed in are shown in Table 4 and 5.

Table 3. Market segments of three-phase asynchronous motor

\begin{tabular}{cccccc}
\hline Market Segment & $\begin{array}{c}\text { Rated Power } \\
(\mathrm{kW})\end{array}$ & $\begin{array}{c}\text { Rotation Speed } \\
(\mathrm{r} / \mathrm{min})\end{array}$ & $\begin{array}{c}\text { Market Segment } \\
\begin{array}{c}\text { Rated Power } \\
(\mathrm{kW})\end{array}\end{array}$ & $\begin{array}{c}\text { Rotation Speed } \\
(\mathrm{r} / \mathrm{min})\end{array}$ \\
\hline$m_{1}$ & 3 & {$[3000,2800]$} & $m_{11}$ & 15 & {$[1000,950]$} \\
$m_{2}$ & 3 & {$[1500,1400]$} & $m_{12}$ & 15 & {$[750,700]$} \\
$m_{3}$ & 3 & {$[1000,950]$} & $m_{13}$ & 22 & {$[3000,2800]$} \\
$m_{4}$ & 3 & {$[750,700]$} & $m_{14}$ & 22 & {$[1500,1400]$} \\
$m_{5}$ & 7.5 & {$[3000,2800]$} & $m_{15}$ & 22 & {$[1000,950]$} \\
$m_{6}$ & 7.5 & {$[1500,1400]$} & $m_{16}$ & 22 & {$[750,700]$} \\
$m_{7}$ & 7.5 & {$[1000,950]$} & $m_{17}$ & 30 & {$[3000,2800]$} \\
$m_{8}$ & 7.5 & {$[750,700]$} & $m_{18}$ & 30 & {$[1500,1400]$} \\
$m_{9}$ & 15 & {$[3000,2800]$} & $m_{19}$ & 30 & {$[1000,950]$} \\
$m_{10}$ & 15 & {$[1500,1400]$} & $m_{20}$ & 30 & {$[750,700]$} \\
\hline
\end{tabular}

Table 4. Segments and their market shares

\begin{tabular}{lcccccccccc}
\hline Market Segment & $m_{1}$ & $m_{2}$ & $m_{3}$ & $m_{4}$ & $m_{5}$ & $m_{6}$ & $m_{7}$ & $m_{8}$ & $m_{9}$ & $m_{10}$ \\
\hline Market Share & $11 \%$ & $7 \%$ & $3 \%$ & $2 \%$ & $7 \%$ & $6 \%$ & $4 \%$ & $3 \%$ & $8 \%$ & $7 \%$ \\
\hline
\end{tabular}

Table 5. Segments and their market shares (Cont.)

\begin{tabular}{lcccccccccc}
\hline Market Segment & $m_{11}$ & $m_{12}$ & $m_{13}$ & $m_{14}$ & $m_{15}$ & $m_{16}$ & $m_{17}$ & $m_{18}$ & $m_{19}$ & $m_{20}$ \\
\hline Market Share & $4 \%$ & $4 \%$ & $7 \%$ & $5 \%$ & $3 \%$ & $2 \%$ & $7 \%$ & $5 \%$ & $3 \%$ & $2 \%$ \\
\hline
\end{tabular}

\subsection{DTPs and PRPs}

Three-phase asynchronous motors possess dozens of DTPs. In this paper, some of the most representative design parameters are selected, including slot number of each phase $d_{1}$, pole number $d_{2}$, plane height $d_{3}$, rotor slot number $d_{4}$, stator inner diameter $d_{5}$, stator outer 
diameter $d_{6}$, iron core length $d_{7}$ and gap length $d_{8}$. Among these parameters, $d_{1}$ is a common parameter, and its value is invariable, while others are scalable design parameters. The weights of these DTPs can be computed by pair-wise comparison as listed in Table 6.

Table 6. Weights of DTPs

\begin{tabular}{ccccccccc}
\hline & $d_{1}$ & $d_{2}$ & $d_{3}$ & $d_{4}$ & $d_{5}$ & $d_{6}$ & $d_{7}$ & $d_{8}$ \\
\hline Weigh & 0.14 & 0.40 & 0.14 & 0.06 & 0.06 & 0.14 & 0.03 & 0.03 \\
\hline
\end{tabular}

Meanwhile, there are five PRPs: rated efficiency $g_{1}$, rated power factor $g_{2}$, starting current ratio $g_{3}$, starting torque ratio $g_{4}$, and maximum torque ratio $g_{5}$. These performance parameters are proportional to the customer's satisfaction except for $g_{3}$ which is inversely proportional to the customer's satisfaction. This is because a larger starting current ratio will result in over-heating, declination of lifespan and shock of the power grid, yet a smaller starting current ratio is a better choice for customers. There are 20 alternative product variants in the product family, the values of the DTPs and PRPs are listed in Table 7.

Table 7. The values of DTPs and PRPs of alternative variants

\begin{tabular}{cccccccccccccc}
\hline & $d_{1}$ & $d_{2}$ & $d_{3}$ & $d_{4}$ & $d_{5}$ & $d_{6}$ & $d_{7}$ & $d_{8}$ & $g_{1}$ & $g_{2}$ & $g_{3}$ & $g_{4}$ & $g_{5}$ \\
\hline$P_{1}$ & 4 & 2 & 100 & 20 & 94 & 155 & 100 & 0.3 & 82 & 0.87 & 7 & 2.2 & 2.2 \\
$P_{2}$ & 4 & 4 & 100 & 38 & 98 & 155 & 135 & 0.25 & 82.5 & 0.81 & 7 & 2.2 & 2.2 \\
$P_{3}$ & 4 & 6 & 132 & 44 & 148 & 210 & 110 & 0.35 & 83 & 0.76 & 6.5 & 2.0 & 2.0 \\
$P_{4}$ & 4 & 8 & 132 & 60 & 148 & 210 & 140 & 0.35 & 82 & 0.72 & 5.5 & 2.0 & 2.0 \\
$P_{5}$ & 4 & 2 & 132 & 20 & 116 & 210 & 125 & 0.55 & 86.2 & 0.88 & 7 & 2.2 & 2.2 \\
$P_{6}$ & 4 & 4 & 132 & 38 & 136 & 210 & 160 & 0.4 & 87 & 0.85 & 7 & 2.2 & 2.2 \\
$P_{7}$ & 4 & 6 & 160 & 58 & 180 & 260 & 145 & 0.4 & 86 & 0.78 & 6.5 & 2.0 & 2.0 \\
$P_{8}$ & 4 & 8 & 160 & 60 & 180 & 260 & 195 & 0.4 & 86 & 0.75 & 5.5 & 2.0 & 2.0 \\
$P_{9}$ & 4 & 2 & 160 & 20 & 150 & 260 & 155 & 0.65 & 88.2 & 0.88 & 7 & 2.0 & 2.2 \\
$P_{10}$ & 4 & 4 & 160 & 38 & 170 & 260 & 195 & 0.5 & 88.5 & 0.85 & 7 & 2.2 & 2.2 \\
$P_{11}$ & 4 & 6 & 180 & 58 & 205 & 290 & 200 & 0.45 & 89.5 & 0.81 & 6.5 & 1.8 & 2.0 \\
$P_{12}$ & 4 & 8 & 200 & 82 & 230 & 327 & 195 & 0.5 & 88 & 0.76 & 6 & 1.8 & 2.0 \\
$P_{13}$ & 4 & 2 & 180 & 20 & 160 & 290 & 175 & 0.8 & 89 & 0.89 & 7 & 2.0 & 2.2 \\
$P_{14}$ & 4 & 4 & 180 & 44 & 187 & 290 & 220 & 0.55 & 91.5 & 0.86 & 7 & 2.0 & 2.2 \\
$P_{15}$ & 4 & 6 & 200 & 58 & 230 & 327 & 220 & 0.5 & 90.2 & 0.83 & 6.5 & 1.8 & 2.0 \\
$P_{16}$ & 4 & 8 & 225 & 82 & 260 & 368 & 310 & 0.5 & 90 & 0.78 & 6 & 1.8 & 2.0 \\
$P_{17}$ & 4 & 2 & 200 & 20 & 182 & 327 & 180 & 1 & 90 & 0.89 & 7 & 2.0 & 2.2 \\
$P_{18}$ & 4 & 4 & 200 & 44 & 210 & 327 & 230 & 0.65 & 92.2 & 0.87 & 7 & 2.0 & 2.2 \\
$P_{19}$ & 4 & 6 & 225 & 60 & 260 & 368 & 210 & 0.5 & 90.2 & 0.85 & 6.5 & 1.7 & 2.0 \\
$P_{20}$ & 4 & 8 & 250 & 82 & 285 & 400 & 225 & 0.55 & 90.5 & 0.80 & 6 & 1.8 & 2.0 \\
\hline & & & & & & & & & & & &
\end{tabular}

Each alternative variant in the product family will face some competitors in the same market segment. The choice probability of each product reflects the competitive result of this customer group. Take customer group $\mathrm{m}_{1}$ as an example:

Table 8 lists the PRPs of all products in market segment $m_{1}$. In this group, company product $P_{1}$ participates in the competition with five other competitors (i.e. $P_{c 1} \sim P_{c 5}$ ).

It can be seen from Table 8 that $P_{c 4}$ has the best performance, and possess the highest PFCA among all of these products. Accordingly, according to Eq.(1), for customer performance parameter $g_{1}$ (rated efficiency) of product $P_{1}$, its customer satisfaction level $u_{11}$ can be calculated as follows:

$$
u_{11}=\frac{0.82-0.80}{0.825-0.80}=0.8
$$

Also, according to Eq. (2), the customer satisfaction level $u_{13}$ of $g_{3}$ (starting current ratio) of $P_{1}$ is calculated as follows: 


$$
u_{13}=\frac{7.3-7}{7.3-6.9}=0.75
$$

Similarly, the customer satisfaction levels of other PRPs are calculated.
Assuming the weights of the PRPs are 0.25, $0.25,0.2,0.2$ and 0.1 , respectively. According to Eq. (3), the customer satisfaction level $U_{1}$ is 0.8 . Table 9 lists of satisfaction levels of all the products in market segment $m_{1}$.

Table 8. PRPs of all products in the market segment $m_{1}$

\begin{tabular}{cccccc}
\hline & $g_{1}$ & $g_{2}$ & $g_{3}$ & $g_{4}$ & $g_{5}$ \\
\hline$P_{1}$ & 0.82 & 0.87 & 7 & 2.2 & 2.2 \\
$P_{c 1}$ & 0.82 & 0.86 & 7.3 & 2.2 & 2.2 \\
$P_{c 2}$ & 0.80 & 0.84 & 7.3 & 2.1 & 2.0 \\
$P_{c 3}$ & 0.81 & 0.85 & 7.2 & 2.1 & 2.1 \\
$P_{c 4}$ & 0.825 & 0.87 & 6.9 & 2.3 & 2.2 \\
$P_{c 5}$ & 0.81 & 0.86 & 7 & 2.1 & 2.2 \\
\hline
\end{tabular}

Table 9. Customer satisfaction levels of all the products in market segment $m_{1}$

\begin{tabular}{ccccccc}
\hline & $g_{1}$ & $g_{2}$ & $g_{3}$ & $g_{4}$ & $g_{5}$ & $U_{i}$ \\
\hline$P_{1}$ & 0.80 & 1.00 & 0.75 & 0.50 & 1.00 & 0.80 \\
$P_{c 1}$ & 0.80 & 0.66 & 0.00 & 0.50 & 1.00 & 0.56 \\
$P_{c 2}$ & 0.00 & 0.00 & 0.00 & 0.00 & 0.00 & 0.00 \\
$P_{c 3}$ & 0.40 & 0.33 & 0.25 & 0.00 & 0.50 & 0.28 \\
$P_{c 4}$ & 1.00 & 1.00 & 1.00 & 1.00 & 1.00 & 1 \\
$P_{c 5}$ & 0.40 & 0.66 & 0.75 & $0 . .00$ & 1.00 & 0.52 \\
\hline
\end{tabular}

After obtaining overall customer satisfaction levels of each product in a market segment, customer choice probabilities of the products can

$$
C_{1}=\frac{e^{2.5 \times U_{1}}}{e^{2.5 \times U_{1}}+\sum_{n=2}^{6} e^{2.5 \times U_{n}}}=\frac{e^{2.5 \times 0.8}}{e^{2.5 \times 0.8}+\left(e^{2.5 \times 0.56}+e^{2.5 \times 0.00}+e^{2.5 \times 0.28}+e^{2.5 \times 1}+e^{2.5 \times 0.52}\right)}=24.3 \%
$$

Similarly, all customer choice probabilities of all products in the market segment $m_{1}$ can be obtained as shown in Table 10. Therefore, the customer selection probabilities of 20 alternative be measured. Set $\lambda=2.5$, then the choice probability of $P_{1}$ is calculated as follows:

Table 10. customer choice probabilities of all products in the market segment $m_{1}$

\begin{tabular}{ccccccc}
\hline & $P_{1}$ & $P_{c 1}$ & $P_{c 2}$ & $P_{c 3}$ & $P_{c 4}$ & $P_{c 5}$ \\
\hline Choice probability & $24.3 \%$ & $13.6 \%$ & $3.3 \%$ & $6.7 \%$ & $40.1 \%$ & $12.0 \%$ \\
\hline
\end{tabular}

Table 11. Customer selection probabilities of 20 alternative product variants

\begin{tabular}{ccccccccccc}
\hline & $P_{1}$ & $P_{2}$ & $P_{3}$ & $P_{4}$ & $P_{5}$ & $P_{6}$ & $P_{7}$ & $P_{8}$ & $P_{9}$ & $P_{10}$ \\
\hline Choice probability & $24.3 \%$ & $5 \%$ & $35 \%$ & $30 \%$ & $25 \%$ & $5 \%$ & $30 \%$ & $5 \%$ & $30 \%$ & $25 \%$ \\
\hline
\end{tabular}

Table 12. Customer selection probabilities of 20 alternative product variants (Cont.)

\begin{tabular}{ccccccccccc}
\hline & $P_{11}$ & $P_{12}$ & $P_{13}$ & $P_{14}$ & $P_{15}$ & $P_{16}$ & $P_{17}$ & $P_{18}$ & $P_{19}$ & $P_{20}$ \\
\hline Choice probability & $15 \%$ & $20 \%$ & $30 \%$ & $25 \%$ & $35 \%$ & $15 \%$ & $40 \%$ & $35 \%$ & $10 \%$ & $10 \%$ \\
\hline
\end{tabular}


In this case, we assume that the profit of each product in the product family is approximately the same, and the profit ratios $\left(o_{i}\right)$ of these 20 products are all 0.05 .

\subsection{Results analysis}

To obtain PSS, GA is applied in this case. The length of chromosome is 20 , and the genes in chromosomes are either 1 or 0 , which indicates the product is selected or not. Moreover, the size of population $\left(n_{p o p}\right)$ is set as 200 , the maximum iteration loop $\left(h_{\max }\right)$ is 100 , and the maximum size of PSS $\left(n_{p s s}\right)$ is 50 . Crossover probability $n_{c}$ and mutation probability $n_{m}$ are 0.8 and 0.01 , respectively.

According to Fig. 2, after 90 iteration loops, there are 44 solutions in PSS as shown in Fig. 2. From the Fig. 3, we can see that more solutions have higher PFCA. However, their commonality (CI) is low. According to Eq. (12), after the comprehensive optimal selection, the optimal solutions can be selected in Table 13 .

Table 13. Solutions with higher priority

\begin{tabular}{|c|c|c|c|c|}
\hline Priority & PFCA & $\mathrm{CI}$ & $\begin{array}{l}\text { The count of } \\
\text { products in solution }\end{array}$ & Product portfolio in solution \\
\hline 1 & 0.22574 & 0.21155 & 17 & $\begin{array}{c}P_{1}, P_{2}, P_{3}, P_{4}, P_{5}, P_{6}, P_{7}, P_{8}, P_{9}, P_{10}, P_{11}, P_{12}, P_{13}, \\
P_{14}, P_{15}, P_{17}, P_{18}\end{array}$ \\
\hline 2 & 0.22224 & 0.22101 & 16 & $\begin{array}{c}P_{1}, P_{3}, P_{4}, P_{5}, P_{6}, P_{7}, P_{8}, P_{9}, P_{10}, P_{11}, P_{12}, P_{13}, P_{14}, \\
P_{15}, P_{17}, P_{18}\end{array}$ \\
\hline 3 & 0.23174 & 0.19252 & 19 & $\begin{array}{c}P_{1}, P_{2}, P_{3}, P_{4}, P_{5}, P_{6}, P_{7}, P_{8}, P_{9}, P_{10}, P_{11}, P_{12}, P_{13}, \\
P_{14}, P_{15}, P_{16}, P_{17}, P_{18}, P_{19}\end{array}$ \\
\hline 4 & 0.22874 & 0.20067 & 18 & $\begin{array}{c}P_{1}, P_{2}, P_{3}, P_{4}, P_{5}, P_{6}, P_{7}, P_{8}, P_{9}, P_{10}, P_{11}, P_{12}, P_{13}, \\
P_{14}, P_{15}, P_{17}, P_{18}, P_{19}\end{array}$ \\
\hline 5 & 0.22424 & 0.21398 & 16 & $\begin{array}{c}P_{1}, P_{2}, P_{3}, P_{4}, P_{5}, P_{6}, P_{7}, P_{9}, P_{10}, P_{11}, P_{12}, P_{13}, P_{14}, \\
P_{15}, P_{17}, P_{18}\end{array}$ \\
\hline
\end{tabular}

The optimal solution with priority 1 has 17 variants and its portfolio is $\left[P_{1}, P_{2}, P_{3}, P_{4}, P_{5}, P_{6}\right.$ $\left., P_{7}, P_{8}, P_{9}, P_{10}, P_{11}, P_{12}, P_{13}, P_{14}, P_{15}, P_{17}, P_{18}\right]$. From the Pareto solutions in this case, we can see that CI has a downward trend with the enhancement of PFCA because of the increasing number of product variants. Meanwhile, different combinations within the same number of product variants have different effects on the solutions. Taking the second and the fifth priority combinations as an example, although they both have 16 product variants, the second solution chooses $P_{8}$ instead of $P_{2}$, and it possesses a larger $\mathrm{CI}$. The fifth one chooses $P_{2}$ instead of $P_{8}$, so the solution has a better PFCA.

The comprehensive optimal selection method is an equal weight method. Thus, the priority given in Table 13 is under the premise that PFCA and CI have the same weight. Companies may put much more emphasis on PFCA if a market is in a depressed or oversupply situation, and more products with higher PFCA will allow companies to obtain more orders. However, when the market becomes more prosperous or in a shortage, companies may put more emphasis on CI. Under this circumstance, companies pursue the maximization of internal efficiency. As a result, there will be fewer product variants in the product family by increasing the weight of $\mathrm{CI}$ in the optimal selection.

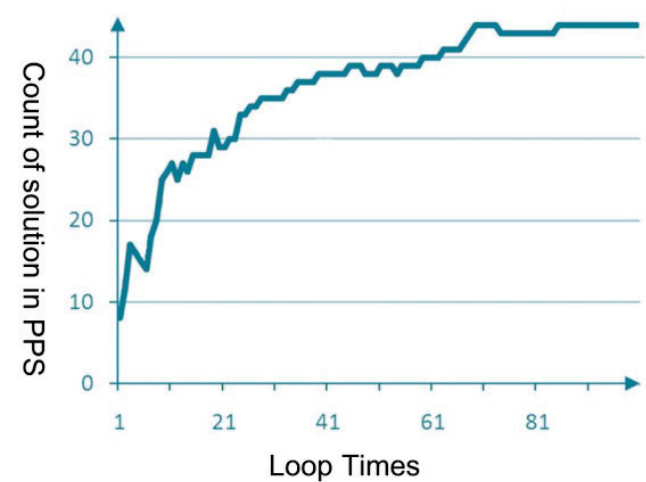

Fig. 2 Iteration process

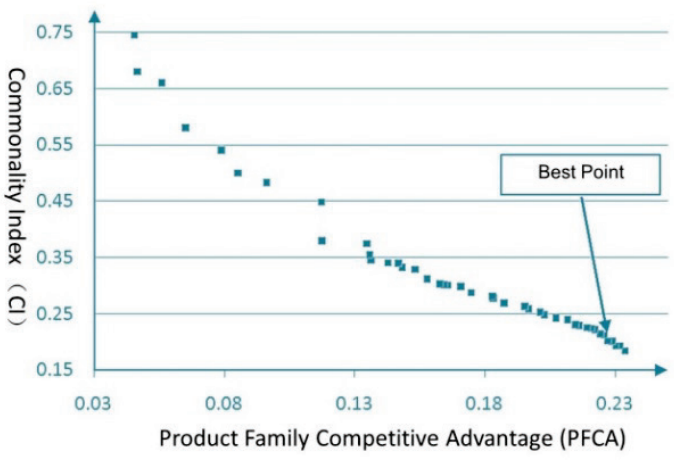

Fig. 3 Pareto optimal solution set 


\subsection{Algorithm feature analysis}

(1) In the case study, the possible solution space reaches 1048575 (i.e. $2^{20}-1$ ), it is difficult to apply exhaustive search techniques or orthogonal arrays to enumerate different combinations of products. The optimization result indicates that the proposed algorithm works efficiently in searching for near-optimal product portfolio solutions.

(2) When applying GA to multi-objective optimization problem, it is important to build a fitness function to find all non-dominated solutions. If the fitness function with constant weight values is constructed, the search direction is fixed, and it is not easy to obtain a variety of non-dominated solutions. In this paper, the fitness function with random weights is developed. And the optimization result reveals that the Pareto frontier for a product family is captured effectively.

\section{Conclusions}

To stand out in the fierce competitions among current manufacturing industries, companies need to provide a competitive product portfolio for the right market. Positioning method of scale-based product family mainly is essentially a combination optimization of various parameters to tradeoff PFCA and commonality. The metrics of PFCA considering customer choice probability, sales, and profits under a holistic framework is proposed. Meanwhile, commonality is measured according to product DTPs in a product family. Based on the metrics of PFCA and commonality, a multi-objective optimization model balancing external competitive advantages and internal commonality is proposed. For capturing the Pareto solutions for the multi-objective optimization model, a GA cooperating fitness function with random weights is developed. According to the case analysis, the optimization model and algorithm yield quality solutions in a reasonable amount of time. The multinomial logit model is applied in this paper, in which the value of scaling parameter $\lambda$ depends on market analysis. Future research may incorporate customer discrete-choice models in the decision making process.

\section{Acknowledgments}

This research is supported by the National Natural Science Foundation of China (Program No. 51275456).

\section{Reference}

[1]X.H. Meng, Z.H. Jiang, G.Q. Huang. On the module identification for product family development.
The International Journal of Advanced Manufacturing Technology, 35(1-2), (2007), pp.26-40.

[2]J. Veldman, A. Alblas. Managing design variety, process variety and engineering change: a case study of two capital good firms. Research in Engineering Design, 23(4), (2012), pp.269-290.

[3]B.J. Pine. Mass Customization: The new frontier in business competition. (Harvard Business School Press, Boston, MA, 1993)

[4]Y.L. Liu, Z.Y. Zhang, Z.X. Liu. Customized configuration for hierarchical products: component clustering and optimization with PSO. The International Journal of Advanced Manufacturing Technology, 57(9-12), (2011), pp.1223-1233.

[5]T.H. Ho, C.S. Tang. Product variety management: research advances. (Boston: Kluwer Academic Publishers, 1998).

[6]M.H. Meyer, A.P. Lehnerd. The power of product platform: Building value and cost leadership. (New York: Free Press, 1997).

[7]P. Child, R. Diederichs, F.H. Sanders, S. Wisniowski. The management of complexity. Sloan Management Review, 33(1), (1991), pp. 73-80.

[8]J. Jiao, Y. Zhang. Product portfolio planning with customer-engineering interaction. IIE Transactions, 37(9), (2005), pp. 801-814.

[9]T.W. Simpson, Z. Siddique, J. Jiao. Product platform and product family design: Methods and applications. (New York: Springer, 2005).

[10]D.A. Collier. The measurement and operating benefits of component part commonality. Decision Sciences,12(1), (1981), pp.85-96.

[11]H.J. Thevenot, T.W. Simpson. Commonality indices for product family design: A detailed comparison. Journal of Engineering Design, 17(2), (2006), pp.99-119.

[12]X. Du, J. Jiao, M.M. Tseng. Architecture of product family: fundamentals and methodology. Concurrent Engineering: Research and Application, 9(4), (2001), pp.309-325.

[13]F. Gao, G. Xiao, T.W. Simpson. Module-scale-based product platform planning. Research in Engineering Design, 20(2), (2009), pp.129-141.

[14]T.W. Simpson, J.R.A. Maier, F. Mistree. Product platform design: Method and application. Research in Engineering Design, 13(1), (2001), pp.2-22.

[15]T.W. Simpson, B.S. D'Souza. Assessing variable levels of platform commonality within a product family using a multi-objective genetic algorithm. Concurrent Engineering Research and Applications, 12(2), (2004), pp.119-130.

[16]J. Jiao, M.M. Tseng. Understanding product family for mass customization by developing commonality indices. Journal of Engineering Design, 11(3), (2000), pp.225-243.

[17]M.V. Martin, K. Ishii. Design for variety: Developing standardized and modularized product platform architectures. Research in Engineering Design, 13(4), (2002), pp.213-235.

[18]T.W. Simpson, C.C. Seepersad, F. Mistree. Balancing commonality and performance within the concurrent design of multiple products in a product family. Concurrent Engineering Research and Applications, 9(3), (2001), pp.177-190.

[19]R. Tian. Research on the scale-based product family design technique and application in the turbo 
expander. (Hangzhou: Zhejiang University, 2008).

$[20] \mathrm{H}$. Li, S. Azarm. An approach for product line design selection under uncertainty and competition. Journal of Mechanical Design. Transactions of the ASME, 124(3), (2002), pp.385-392.

[21]O.L. de Week, E.S. Suh, D. Chang. Product family and platform portfolio optimization. In: Proceedings of the ASME Design Engineering Technical Conferences-Design Automation Conference, Chicago, IL, ASME, Paper No. DETC2003/DAC-48721, 2003.

[22]M.E. PuUmana, W.L. Mooreb, D.G. Wardellb. A comparison of quality function deployment and conjoint analysis in new product design. Journal of Product Innovation Management,19(5), (2002), pp.354-364.

[23]J. Jiao, Y.Y. Zhang, Y. Wang. A heuristic genetic algorithm for product portfolio planning. Computers \& Operations Research, 34(6), (2007), pp.1777-1799.

[24]W.J. Steiner, H. Hruschka. A probabilistic one-step approach to the optimal product line design problem using conjoint and cost data. Review of Marketing Science Working Papers, 1(4), (2002), pp.4 [25]P.E. Green, A.M. Krieger. Models and heuristics for product line selection. Marketing Science, 4(1), (1985), pp.1-19.

[26]P.E. Green, A.M. Krieger. Recent contributions to optimal product positioning and buyer segmentation. European Journal of Operational Research, 41(2), (1989), pp.127-141.

[27] K. Fujita, H. Yoshida. Product variety optimization simultaneously designing module combination and module attributes. Concurrent Engineering: Research and Application, 12(2), (2004), pp.105-118.

[28]S.K. Nair, L.S. Thakur, K. Wen. Near optimal solutions for product line design and selection: beam search heuristics. Management Science, 41(5), (1995), pp.767-785.

[29]A. Kaul, V.R. Rao. Research for product positioning and design decisions: an integrative review. International Journal of Research in Marketing, 12(4), (1995), pp.293 - 320.

[30]S.M. Sait, H. Youssef. Iterative computer algorithms with applications in engineering: solving combinatorial optimization problems. (IEEE Computer Society Press, 1999).

[31]M.R. Garey, D.S. Johnson. Computers and intractability: a guide to the theory of NP-completeness. (New York: Freeman Publisher; 1979).

[32]V. Kreng, T.P. Lee. Modular product design with grouping genetic algorithm: a case study. Computers \& Industrial Engineering, 46(3), (2004), pp.443-460.

[33]C.R. Houck, J.A. Joines, M.G. Kay. Comparison of genetic algorithms, random restart, and two-opt switching for solving large location-allocation problems. Computers \& Operations Research, 23(6), (1996), pp.587 - 96.

[34]P.C. Chu, J.E. Beasley. A genetic algorithm for the generalized assignment problem. Computers \& Operations Research, 24(1), (1997), pp.17-23.

[35]X. Yang, J.J. Song, B.B. Wang, Y.C. Hui. The optimization design of product form and its application based on BP-GA-CBR.In: IEEE 11th International Conference on Computer-Aided Industrial Design and Conceptual Design, CAID and $C D^{\prime} 2010$, Yiwu, China, 2010.

[36]P.E. Green, A.M. Krieger. Individualized hybrid models for conjoint analysis. Management Science, 42(6), (1996), pp.850-867.

[37]I. Syamsuddin, J. Hwang. The Use of AHP in security policy decision making: An open office calc application. Journal of Software, 5(10), (2010), pp.1162-1169.

[38]K. Kumar, S. Agarwal. Multi-objective parametric optimization on machining with wire electric discharge machining. The International Journal of Advanced Manufacturing Technology, 62(5-8), (2012), pp.617-633.

[39]H. Ishibuchi, T. Murata. A multi-objective genetic local search algorithm and its application to flowshop scheduling. IEEE Transactions on Systems, Man, and Cybernetics, Part C: Applications and Reviews, 28(3), (1998), pp.392-403.

[40]E. Zitzler, L. Thiele, M. Laumanns, C.M. Fonseca, V.G. da Fonseca. Performance assessment of multiobjective optimizers: an analysis and review. IEEE Transactions on Evolutionary Computation, 7(2), (2003), pp.117-132.

[41]M.A. Abido. Multi-objective evolutionary algorithms for electric power dispatch problem. IEEE Transactions on Evolutionary Computation, 10(3), (2006), pp.315-329. 